\title{
Another Paradox? A Comment on Lindley (1997)
}

\author{
Fabian Dablander, Don van den Bergh, and Eric-Jan Wagenmakers \\ Department of Psychological Methods, University of Amsterdam
}

\begin{abstract}
Lindley (1997) argued that Bayes factors possess an unsatisfactory, even paradoxical property. In this short comment, we illustrate and clarify Lindley's point with an example. We conclude that said property is not paradoxical, but intuitive.
\end{abstract}

Keywords: Bayes factor, Simpson's paradox

\section{Introduction}

Over twenty years ago, Dennis Lindley argued that Bayes factors possess an unsatisfactory, even paradoxical property (Lindley, 1997, p. 188-189). According to Lindley, Bayes factor proponents could find themselves simultaneously saying that (a) it is more likely to be a tall man than a tall woman, (b) it is more likely to be a short man than a short women, and (c) it is more likely to be a woman than a man. Lindley's judgement is severe: "one hardly advances the respect with which statisticians are held in society by making such declarations." The potential ramifications of Lindley's critique are profound, as the Bayes factor is often considered a valuable measure of statistical evidence. Nevertheless, Lindley's critique has never been addressed in the subsequent literature. As Lindley points out, the scenario at hand is conceptually related to Simpson's paradox. We illustrate the supposed paradox on a toy example, and show that the Bayes factor does not lead to inconsistent statements such as the one outlined by Lindley. Our arguments are two-fold. First, Lindley's statement concerns posterior probabilities, and thus does not apply to Bayes factors. Second, the Bayes factor does admit a reversal, but this reversal is both intuitive and desirable.

\section{A Paradox?}

While Lindley's treatment is abstract and entirely general, it helps to showcase the supposed paradox with a practical example. Assume that two boys, Jim and John, and two girls, Julia and Jane, enter a prediction contest. Their task is to predict whether it will rain in Amsterdam on their first vacation day. We model the outcome as a Bernoulli trial governed by the parameter $\theta$. For their predictions the contenders have to rely solely on their prior beliefs, which for simplicity we assume are adequately expressed as uniform distributions. Specifically, assume

$$
\begin{aligned}
p(\theta \mid \operatorname{Jim}) & =\operatorname{Unif}(0.5,1) \\
p(\theta \mid \mathrm{John}) & =\operatorname{Unif}(0.3,0.6) \\
p(\theta \mid \mathrm{Julia}) & =\operatorname{Unif}(0.4,0.8) \\
p(\theta \mid \mathrm{Jane}) & =\operatorname{Unif}(0.1,0.7),
\end{aligned}
$$

and where, if we specify mixture weights $\pi_{1}=p$ (John $\mid$ Boys) and $\pi_{1}=p($ Julia $\mid$ Girls $)$, and priors over gender $\pi_{B}=p$ (Boys), $\pi_{G}=1-\pi_{B}$, we can combine the priors and thus predictions of the girls and boys. These weights may be given the interpretation of prior probabilities, such that $\pi_{1}$ gives the probability that John's prediction is correct, given that we know that the Boys' predictions is correct, while $\pi_{B}$ gives the overall prior probability that the Boys' predictions is correct. Suppose we take $\pi_{1}=0.2, \pi_{2}=0.8$, and $\pi_{B}=0.5$, then Figure 1 displays the individual and combined priors. 

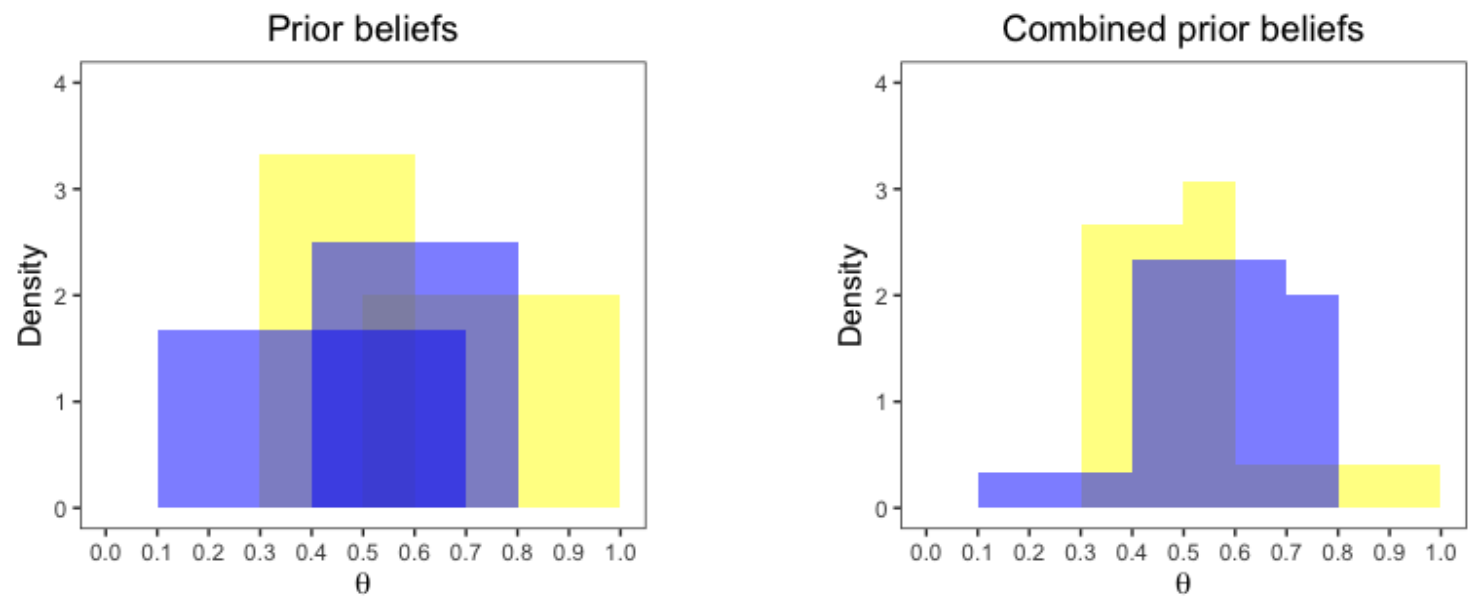

Figure 1: Left panel: prior beliefs of Jim and John (yellow colours) and Julia and Jane (blue colours). Right panel: combined prior beliefs for mixture weights $\pi_{1}=0.2$ and $\pi_{2}=0.8$, and $\pi_{B}=.5$.

Fast forward into the future, we observe that it did in fact rain on their first vacation day, $y=1$. The average predictive success, that is, the marginal likelihood, is given by the mean of the uniform priors; for example

$$
p(y \mid \operatorname{Jim})=\int_{0}^{1} \theta^{y}(1-\theta)^{1-y} \cdot p(\theta \mid \operatorname{Jim}) \mathrm{d} \theta=\int_{0}^{1} \theta \cdot p(\theta \mid \operatorname{Jim}) \mathrm{d} \theta=\frac{1}{2}(1+0.5)=0.75 .
$$

The Bayes factor is a ratio of marginal likelihoods, and thus the Bayes factors in favor of Jim compared to Julia, and John compared to Jane are $\mathrm{BF}_{\mathrm{Jim}>\mathrm{Julia}}=\frac{0.75}{0.60}=1.25$ and $\mathrm{BF}_{\mathrm{John}>\mathrm{Jane}}=$ $\frac{0.45}{0.40}=1.125$, respectively. However, the Bayes factor for the boys compared to the girls also considers the mixture weights, and thus gives

$$
\mathrm{BF}_{\text {Boys }>\text { Girls }}=\frac{\pi_{1} \cdot p(y \mid \mathrm{Jim})+\left(1-\pi_{1}\right) \cdot p(y \mid \mathrm{John})}{\pi_{2} \cdot p(y \mid \mathrm{Julia})+\left(1-\pi_{2}\right) \cdot p(y \mid \mathrm{Jane})}=\frac{\frac{1}{5} \cdot 0.75+\frac{4}{5} \cdot 0.45}{\frac{4}{5} \cdot 0.60+\frac{1}{5} \cdot 0.40}=0.89
$$

This is the "paradox" Lindley bemoans: there is evidence that Jim outpredicts Julia, that John outpredicts Jane, and yet the girls outpredict the boys. Lindley then notes that such a reversal can never happen when posterior odds are employed instead. In our example, the individual posterior odds are

$$
\begin{aligned}
& \mathrm{PO}_{\text {Jim }>\text { Julia }}=\frac{\pi_{\mathrm{B}}}{\pi_{\mathrm{G}}} \cdot \frac{0.2}{0.8} \cdot \mathrm{BF}_{\text {Jim }>\text { Julia }}=0.31 \\
& \mathrm{PO}_{\text {John }>\text { Jane }}=\frac{\pi_{\mathrm{B}}}{\pi_{\mathrm{G}}} \cdot \frac{0.8}{0.2} \cdot \mathrm{BF}_{\text {John }>\text { Jane }}=0.28 \\
& \mathrm{PO}_{\text {Boys }>\text { Girls }}=\frac{\pi_{\mathrm{B}}}{\pi_{\mathrm{G}}} \cdot \mathrm{BF}_{\text {Boys }>\text { Girls }}=0.89,
\end{aligned}
$$

which does not show the reversal: all comparisons favour the girls over the boys both individually and as a group. That such a reversal can never happen when considering posterior odds is apparent by noting that if $\frac{p\left(\mathcal{M}_{1}\right)}{p\left(\mathcal{M}_{2}\right)} \cdot \frac{\pi_{1} \cdot p\left(y \mid \mathcal{M}_{11}\right)}{\pi_{2} \cdot p\left(y \mid \mathcal{M}_{21}\right)}>1$ and $\frac{p\left(\mathcal{M}_{1}\right)}{p\left(\mathcal{M}_{2}\right)} \cdot \frac{\left(1-\pi_{1}\right) \cdot p\left(y \mid \mathcal{M}_{12}\right)}{\left(1-\pi_{2}\right) \cdot p\left(y \mid \mathcal{M}_{22}\right)}>1$, then $\frac{p\left(\mathcal{M}_{1}\right)}{p\left(\mathcal{M}_{2}\right)} \cdot \frac{\pi_{1} \cdot p\left(y \mid \mathcal{M}_{11}\right)+\left(1-\pi_{1}\right) \cdot p\left(y \mid \mathcal{M}_{21}\right)}{\pi_{2} \cdot p\left(y \mid \mathcal{M}_{21}\right)+\left(1-\pi_{2}\right) \cdot p\left(y \mid \mathcal{M}_{22}\right)}>1$ for any individual models $\mathcal{M}_{11}, \mathcal{M}_{21}, \mathcal{M}_{12}$, and $\mathcal{M}_{22}$, and composite models $\mathcal{M}_{1}=\mathcal{M}_{11} \cup \mathcal{M}_{21}, \mathcal{M}_{2}=\mathcal{M}_{12} \cup \mathcal{M}_{22}{ }^{1}$

\section{Assessment and Conclusion}

Lindley's claim that the Bayes factor admits logical inconsistencies such as (a) it is more likely to be a tall man than a tall woman, (b) it is more likely to be a short man than a short women, and

\footnotetext{
${ }^{1}$ This is Lindley's notation.
} 


\begin{tabular}{c|cc} 
& Men & Women \\
\hline Tall & $\frac{9}{10}(90 \%)$ & $\frac{40}{50}(80 \%)$ \\
Short & $\frac{41}{90}(45.5 \%)$ & $\frac{20}{50}(40 \%)$ \\
Total & $\frac{50}{100}(50 \%)$ & $\frac{60}{100}(60 \%)$
\end{tabular}

Table 1: Simpson's reversal: the proportion of tall and short men can be higher than the proportion of tall and short women, respectively, while at the same time the proportion of women is larger than the proportion of men.

(c) it is more likely to be a woman than a man, we believe is incorrect. Even though the word "likely" requires careful inspection - as it can relate to likelihoods as well as probabilities - we believe Lindley's statement is about posterior probabilities, not about Bayes factors. He is correct in stating that it is possible to reverse the Bayes factor, but the interpretation of this reversal changes: it seizes to be illogical but becomes intuitive.

To rephrase Lindley's charge, Bayes factor proponents can find themselves simultaneously saying that (a) the data support the proposition that there are more tall men than tall women, (b) the data support the proposition that there are more short men than short women, and (c) the data support the proposition that there are more women than men. In our example, this reversal means that while Jim outpredicts Julia and John outpredicts Jane, a specific weighted linear combination of Jim and John's predictions is worse than a specific weighted linear combination of Julia and Jane's prediction. If we were to repeat this prediction contest many times, these weights would correspond to having John predict more frequently than Jim, and Julia more frequently than Jane. As Jane outpredicts Jim, the reversal occurs. ${ }^{2}$.

Lindley argues that this property of Bayes factors relates to Simpson's paradox, but this claim can be made more precise. To do so, we follow Pearl (2014) and distinguish between Simpson's reversal and Simpson's paradox. Simpson's reversal is simply a property of fractions, as illustrated in Table 1. Simpson's paradox, on the other hand, relates to the fact that the exact same data may be analyzed either on the level of the whole population or on the level of the subpopulations. The correct analysis depends on the causal story about how the data have been generated. We believe that the situation with Bayes factors is analogous to Simpson's reversal, not to Simpson's paradox. As the former is a property of fractions, one can always choose the fractions in a way such that Simpson's reversal occurs. Analogously, one can always choose the mixture weights within the composite models in such a way that the Bayes factor reversal occurs - except when every submodel of $\mathcal{M}_{1}$ outpredicts every submodel of $\mathcal{M}_{2}$. In a sense, this dependency merely means that the answer depends on the question and prior specification. Are we interested in whether the boys individually outpredict the girls? Then condition. Are we interested in whether the boys jointly outpredict the girls? Then marginalise.

What is commonly referred to "Lindley's paradox" exposed a deep philosophical divide between frequentist and Bayesian statistics, and helped emphasize the perils of uninformative priors for Bayesian hypothesis testing (Lindley, 1957; DeGroot, 1982). The Bayes factor proponents among us may consider themselves lucky: what initially seemed like a damning critique by one of the fields most brilliant scholars turned out to expose not a paradoxical, but an intuitive property of Bayes factors. Thus, although Lindley's original critique lost its force, it nevertheless illuminates an important difference between the Bayes factor and posterior odds. In this spirit — by explicating and carefully analysing Lindley's critique - we hope that our short comment has been useful.

\section{References}

DeGroot, M. H. (1982). Lindley's paradox: Comment. Journal of the American Statistical Association, $77(378)$, 336-339.

Lindley, D. V. (1957). A statistical paradox. Biometrika, 44(1/2), 187-192.

\footnotetext{
${ }^{2}$ More generally, a necessary condition for the reversal to occur is that not every individual model in $\mathcal{M}_{1}$ is able to outpredict every individual model in $\mathcal{M}_{2}$.
} 
Lindley, D. V. (1997). Some comments on Bayes factors. Journal of Statistical Planning and Inference, 61(1), 181-189.

Pearl, J. (2014). Comment: Understanding Simpson's paradox. The American Statistician, 68(1), $8-13$. 